\title{
RiskFree - Uma Ferramenta de apoio à Gerência de Riscos em Projetos de Software ${ }^{\mathrm{i}}$
}

\author{
Flávio Knob, Filipi Silveira, Afonso Inácio Orth, Rafael Prikladnicki \\ Pontifícia Universidade Católica do Rio Grande do Sul - PUCRS \\ Av. Ipiranga, 6681 - Prédio 30 - CEP 90.619-900 - Porto Alegre - RS - Brasil \\ $\{$ knob, fpsilveira\}@ $@$ terra.com.br \{orth, rafael $\} @$ inf.pucrs.br
}

\begin{abstract}
In this paper, we present a flexible risk management tool - RiskFree. It is designed to help project managers and their teams to manage, monitor, and control risks in software development projects by easily adapting to the organizational needs. Furthermore, it conforms to the risk management process practices of the CMMI model level three.
\end{abstract}

Keywords: Project Management, Risk Management, PMI, CMMI

Resumo. Neste artigo é apresentada a ferramenta RiskFree. A ferramenta tem como objetivo auxiliar gerentes de projetos e suas equipes a gerenciar riscos em projetos de desenvolvimento de software. Uma das principais características da ferramenta é o fato de poder ser adaptada para contemplar a realidade da organização em que for inserida. Além disso, ela foi desenvolvida de acordo com as práticas e objetivos da área de processo de gerência de riscos do nível três do modelo CMMI.

Palavras-chave: Gerência de Projeto, Gerência de Riscos, PMI, CMMI

\section{Introdução}

A área de gerência de projetos vem recebendo uma atenção cada vez maior por parte das organizações, merecendo assim posição de destaque dentro das mesmas (Prikladnicki et. al., 2005). A prova disso é o crescente número de organizações que aderem à gestão orientada a projetos, ou seja, focada em projetos. Os projetos, por sua vez, a cada dia tornam-se maiores e mais complexos (Demarco \& Lister, 2003).

A idéia de que a gerência de riscos é importante e deve ser integrada à gerência de projetos é consenso entre os gerentes de projetos (Del Caño \& De La Cruz, 2002). Por parte dos executivos, o interesse no assunto nunca foi tão grande, e nunca esteve tão evidente. Porém, são grandes também as dificuldades para compreensão e a implantação efetiva da gerência de riscos. A falta de ferramentas específicas para o gerenciamento de riscos ou mesmo a dificuldade de acesso a estas ferramentas, devido ao seu custo elevado, podem ter agravado esse problema. Existem atualmente no mercado algumas ferramentas voltadas especificamente para a gerência de riscos. No entanto, estas ferramentas são em sua maioria comerciais e, muitas vezes, tem um custo tão elevado que inviabiliza a sua adoção por organizações de pequeno e médio porte.

Este artigo apresenta a RiskFree, uma ferramenta com o objetivo de auxiliar equipes de projetos nas tarefas relacionadas à gerência de riscos em projetos de desenvolvimento de software. Além do desenvolvimento da ferramenta, o foco deste trabalho, gerência de riscos, permitiu que o objeto em estudo fosse visto e desenvolvido com maior profundidade. A ferramenta RiskFree supre, pelo menos em parte, a carência 
de ferramentas específicas na área de gerência de riscos em projetos de software e as necessidades não atendidas pelas ferramentas existentes atualmente. Dentre as necessidades atendidas pode-se citar: uma ferramenta gratuita, em língua portuguesa, adaptável, e atendendo a área de processo de gerência de riscos do modelo CMMI.

O artigo está organizado em 6 seções. A seção 2 apresenta o referencial teórico da gerência de risco. Na seção 3 apresenta-se uma análise comparativa de ferramentas de gerência de risco existentes no mercado. Na seção 4 apresenta-se a descrição da ferramenta RiskFree. Na seção 5 apresentam-se as considerações finais. As referências bibliográficas são apresentadas na seção 6 .

\section{Gerência de riscos em projetos de software}

Risco como ciência nasceu no século XVII, no Renascimento (Bernstein, 1997). Na área de software, o risco foi representado de forma sistemática por Barry Boehm, nos anos 80, através do modelo em espiral, que tem como princípio ser iterativo e dirigido à análise do risco em cada iteração (Boehm, 1991). A palavra "risco" deriva do italiano antigo "risicare", que significa "ousar" (Bernstein, 1997). Risco é uma opção e não um destino. Por isso, requer mais do que processos competentes e uma habilidade de pensar intuitivamente; requer disciplina. A esta disciplina dá-se o nome de gerência de risco.

Atualmente, a gerência de risco na Engenharia de Software é uma evolução do conceito de risco, que passou de uma análise dentro do modelo de desenvolvimento, para uma gerência, que deve permear todo o ciclo de vida do software. Devido a sua característica de produzir algo único, os projetos tornam-se ambientes de incertezas (PMI, 2000). Estas incertezas, inerente aos projetos, é o que faz com que os riscos estejam presentes ao longo do ciclo de vida. E o gerenciamento de risco é um processo pró-ativo, invocado para tentar eliminar problemas potenciais e as incertezas antes que eles ocorram, aumentando a probabilidade de sucesso do projeto (Boehm, 1991).

Segundo (Demarco \& Lister, 2003), organizações que preferem fugir dos riscos, mantendo o foco apenas naquilo em que são especialistas, estão perdendo espaço no mercado. Isto é verdade no sentido de que estas organizações estão deixando de aproveitar novas oportunidades. Segundo (Kruchten, 2003), em um ciclo de vida iterativo, muitas decisões são conduzidas pela análise dos riscos do projeto. Para tomar decisões efetivas é preciso ter uma compreensão clara dos riscos e de estratégias para reduzir o impacto caso os mesmos venham a ocorrer. Por sua vez, (Schwalbe, 2002) diz que, apesar de muitas vezes ser um fator decisivo para que o projeto seja bem sucedido, a gerência de riscos é ainda um aspecto ignorado dentro da gerência de projetos. Segundo a autora, a aplicação de práticas de gerência de riscos traz vantagens tais como:

- Auxílio na seleção de projetos;

- Ajuda a determinar o escopo de projetos;

- Ajuda a desenvolver cronogramas e estimativas de custos realistas;

- Ajuda os interessados do projeto a entenderem a natureza do projeto;

- Torna a equipe do projeto participante da definição de pontos fortes e fracos;

- Ajuda a integrar as demais áreas de conhecimento da gerência de projetos;

Assim como (Demarco \& Lister, 2003), Schwalbe também ressalta que organizações não devem fugir dos riscos, pois podem estar deixando de aproveitar grandes oportunidades. Dado que todos os projetos envolvem riscos e oportunidades, a questão é saber decidir quais projetos são vantajosos para a organização e como os riscos inerentes ao projeto serão gerenciados ao longo do seu ciclo de vida. 


\section{Estudo de Ferramentas de Gerência de Risco ${ }^{\mathrm{ii}}$}

De forma a identificar as características de algumas ferramentas de gerência de riscos existentes no mercado, foram estudadas três ferramentas. Todas elas abordam a gerência de riscos como um todo. Infelizmente, não foram encontradas ferramentas de gerência de risco em língua portuguesa, disponíveis na Internet. O objetivo do estudo foi sustentar de forma mais consistente a necessidade do desenvolvimento da ferramenta proposta. A análise foi feita com base nos seguintes critérios: processo de gerência de risco (fases do processo de gerência de risco contempladas); usabilidade (facilidade de uso); preço (valor de uma licença, caso não seja gratuita); plataforma (quais plataformas são suportadas e existência dependências de outras aplicações); documentação (disponibilidade, relevância e abrangência da documentação); integração (capacidade de integrar-se com outras áreas ou ferramentas de gerência de projeto); relatórios: (disponibilidade de visualização e impressão de relatórios); idiomas suportados: (idiomas disponíveis e a capacidade de suportar novos idiomas).

Todas as informações foram obtidas através dos sites dos desenvolvedores das ferramentas. A veracidade das informações não foi em nenhum momento questionada. Em alguns casos a omissão ou escassez de informações sobre algum recurso ou característica específica foi subentendida como não disponível. A tabela 1 apresenta uma comparação entre as ferramentas analisadas. Nesta tabela foram resumidas e reunidas as características avaliadas na análise individual de cada ferramenta.

Tabela 1 - Tabela comparativa entre as ferramentas analisadas

\begin{tabular}{|l|c|c|c|}
\hline Características & \multicolumn{1}{c|}{ Risk Radar } & \multicolumn{2}{c|}{ RiskTrack } \\
Processo de gerência de & $\begin{array}{c}\text { CMM nível 3, IEEE } \\
\text { Standard 1540, e } \\
\text { outros }\end{array}$ & $\begin{array}{c}\text { Própro - ARM } \\
\text { Assessment Report } \\
\text { Manage) }\end{array}$ & $\begin{array}{c}\text { Não foram } \\
\text { encontradas } \\
\text { informações }\end{array}$ \\
\hline Usabilidade & $\begin{array}{c}\text { Boa, explora bem } \\
\text { recursos visuais }\end{array}$ & $\begin{array}{c}\text { Razoável, não é muito } \\
\text { intuitiva }\end{array}$ & $\begin{array}{c}\text { Razoável, requer } \\
\text { conhecimento de } \\
\text { Excel }\end{array}$ \\
\hline Preço (1 usuário) & US\$795,00 & US\$1.495,00 & US\$685,00 \\
\hline Plataforma & $\begin{array}{c}\text { Microsoft Windows } \\
\text { (requer Microsoft } \\
\text { Access) }\end{array}$ & Microsoft Windows & $\begin{array}{c}\text { Microsoft Windows } \\
\text { (requer Microsoft } \\
\text { Excel) }\end{array}$ \\
\hline Documentação & Sim & Sim & $\begin{array}{c}\text { Sim, em quatro } \\
\text { idiomas (ing, fra, esp, } \\
\text { ale) }\end{array}$ \\
\hline Integração & Não & Não & Não \\
\hline Relatórios & Sim (+ de 20 modelos) & Sim (6 modelos) & Sim (com gráficos) \\
\hline Idiomas & Somente inglês & Somente inglês & Somente inglês \\
\hline
\end{tabular}

Com base na tabela anterior, percebe-se nas ferramentas analisadas a forte dependência da plataforma Microsoft Windows e de aplicativos do pacote Microsoft Office. Percebe-se também que nenhuma das ferramentas está disponível em língua portuguesa e que nenhuma é capaz de integrar-se com outras ferramentas de gerência de projetos ou de gerência de riscos.

\section{A ferramenta RiskFree}

A ferramenta RiskFree foi idealizada e construída com o objetivo de suprir as necessidades citadas na seção 1. A ferramenta se baseou nas principais práticas sugeridas por (PMI, 2000) e (SEI, 2002), que são duas referências amplamente aceitas pelo mercado. Como um dos objetivos era permitir o uso por empresas de pequeno e médio, tomou-se o cuidado de fazer uso apenas de tecnologias baseadas em software livre (a ferramenta foi desenvolvida em J2EE com banco de dados PostgreSQL, distribuídas gratuitamente na Internet). 
No intuito de respeitar as diferenças existentes na realização das atividades de gerência de riscos entre organizações diferentes, a ferramenta foi desenvolvida de forma a permitir que estas diferenças possam ser implementadas e disponibilizadas na forma de componentes. Isto dá à ferramenta uma característica de adaptabilidade, uma vez que os usuários não precisam ficar restritos a um número limitado de funcionalidades.

Para comprovar o funcionamento da arquitetura proposta, bem como para prover um conjunto mínimo de funcionalidades (suficientes para tornar a ferramenta utilizável) foram desenvolvidos cinco componentes, cada um para uma etapa diferente do processo de gerência de riscos. A seguir são apresentados em detalhes o processo de gerência de riscos definido, a característica de adaptabilidade e os componentes desenvolvidos.

\subsection{O processo de gerência de riscos da ferramenta}

$\mathrm{O}$ processo de gerência de risco implementado na ferramenta é muito semelhante ao conjunto de práticas proposto no PMBOK (PMI, 2000), que foi a base para o desenvolvimento. A única diferença é que, na ferramenta, as análises qualitativa e quantitativa previstas no PMBOK foram reunidas em apenas uma atividade de análise.

Cada uma das etapas que compõem o processo de gerência de riscos definido possui objetivos específicos. A seguir são apresentados estes objetivos:

- Planejamento da gerência de riscos: tem como principal objetivo a elaboração do plano de gerência de riscos do projeto. Segundo (PMI, 2000), este plano deve definir como e quando as atividades de identificação, análise, planejamento de resposta, monitoração e controle dos riscos irão ocorrer ao longo do projeto. Alguns outros aspectos como metodologias, papéis e responsabilidades, orçamento e formato de relatórios também podem constar no plano;

- Identificação dos riscos: tem como principal objetivo identificar e classificar os riscos que afetam o projeto. Para cada risco, são identificados também seus sintomas e sinais de alerta. Este processo caracteriza-se por ser iterativo, à medida que o projeto avança novos riscos vão sendo identificados;

- Análise dos riscos: para cada risco identificado deve ser realizada uma atividade de análise que tem como objetivo verificar a probabilidade de ocorrência do risco e o seu impacto em relação aos objetivos do projeto. A atividade de análise é composta pela análise qualitativa, que tem o objetivo de priorizar os riscos de acordo com a sua probabilidade de ocorrência e impacto caso o risco venha a ocorrer, e pela análise quantitativa, que tem o objetivo de analisar numericamente a probabilidade e eventuais conseqüências de cada risco;

- Planejamento de resposta aos riscos: tem como principal objetivo determinar ações para aproveitar as oportunidade e endereçar os riscos do projeto. Esta atividade inclui a atribuição de responsabilidades para cada risco identificado, garantindo um melhor controle sobre os riscos do projeto;

- Monitoração e controle dos riscos: tem como principal objetivo garantir que o plano de gerência de riscos seja seguido e que os riscos identificados e endereçados estejam sob controle. Esta atividade caracteriza-se por ser contínua dentro do ciclo de vida do projeto.

O objetivo principal da ferramenta desenvolvida é prover uma ferramenta para auxiliar a equipe de projeto nas etapas que compõem o processo de gerência de riscos. Para isto, a ferramenta disponibiliza algumas das práticas e técnicas propostas por (PMI, 2000) como, por exemplo: um facilitador para a elaboração de um plano de gerência de 
riscos, estrutura analítica de riscos, matriz de probabilidade e impacto, acompanhamento da situação dos riscos entre outras. A Figura 1 apresenta o processo de gerência de riscos que foi implementado na ferramenta.

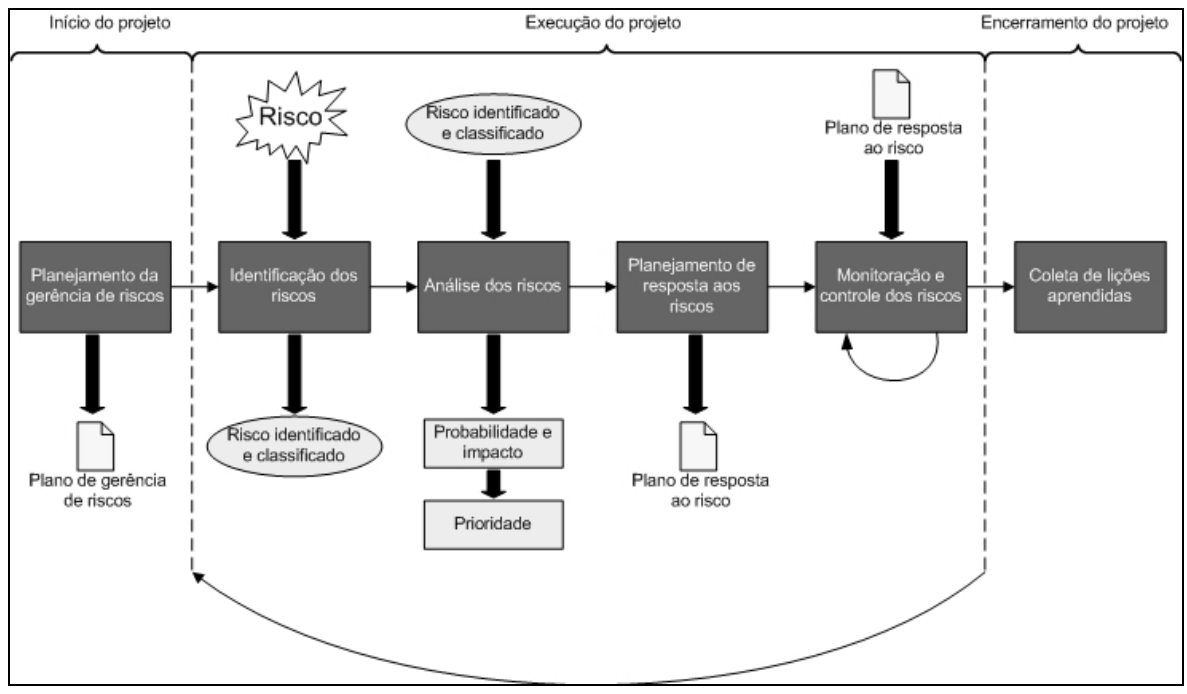

Figura 1 - Processo de gerência de riscos implementado

De forma complementar ao processo definido, a ferramenta permite a implementação do conceito de aprendizagem. O motivo pelo qual se decidiu por contemplar este conceito no processo é que várias das técnicas e ferramentas utilizadas nos processos citados anteriormente fazem uso de informações históricas, provenientes de projetos passados, para gerar resultados mais apurados.

\subsection{Adaptabilidade}

Para cada uma das etapas que compõem o processo de gerência de riscos definido, existem diversas práticas e técnicas que auxiliam a atingir seus respectivos objetivos. Segundo (PMI, 2000), alguns exemplos são:

- Planejamento da gerência de riscos: reuniões de planejamento;

- Identificação dos riscos: revisão da documentação, coleta de informações, listas de verificação, análises das premissas e técnicas de diagramação;

- Análise dos riscos: probabilidade e impacto dos riscos, matriz de classificação da probabilidade/impacto dos riscos, teste das premissas do projeto, precisão dos dados, entrevistas, análise sensitiva, análise de árvores de decisão e simulação;

- Planejamento de resposta aos riscos: prevenção, transferência, mitigação, etc.;

- Monitoração e controle dos riscos: auditorias de resposta aos riscos, revisões periódicas dos riscos do projeto, análise do valor agregado, medição técnica do desempenho e planejamento adicional de resposta aos riscos;

- Coleta de lições aprendidas: reuniões de post mortem.

Para permitir que cada organização pudesse utilizar as técnicas que melhor atendessem às suas necessidades (o que pode incluir até mesmo técnicas próprias), a ferramenta foi construída de forma que fosse possível vincular componentes a cada etapa do processo de gerência de riscos definido. Desta forma, a organização que fizer uso da ferramenta não fica restrita a um conjunto limitado e pré-definido de técnicas. A Figura 2 apresenta a arquitetura de componentes da ferramenta, que é formada por: 
- Hibernate: framework de persistência de dados utilizado no projeto;

- RiskFreeCore: provê funcionalidades comuns a todos os componentes, incluindo as de acesso à dados e de autorização;

- RiskFreeMain: provê funcionalidades de administração e configuração;

- RiskFreeComponent: implementação das técnicas e ferramentas envolvidas no processo de gerência de riscos.

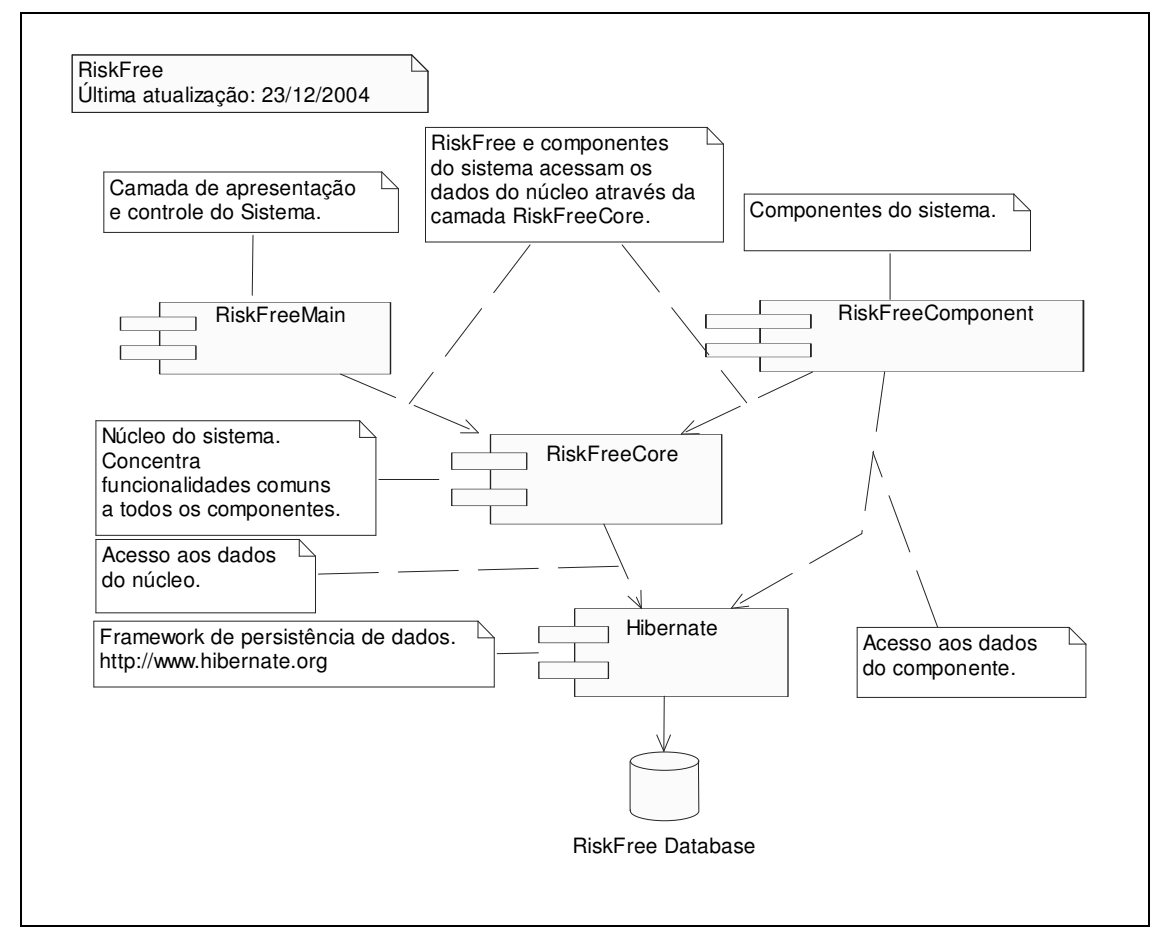

Figura 2 - Arquitetura de componentes da ferramenta RiskFree

Os componentes desenvolvidos são instalados e vinculados a algum dos pontos de adaptação da ferramenta, que atualmente compreendem as etapas do processo de gerência de riscos; as informações gerais e sumarizadas sobre o projeto; e os relatórios em nível de projeto e organizacional.

\subsection{Os componentes desenvolvidos}

Aproveitou-se a característica de adaptabilidade da ferramenta para desenvolver componentes que atendessem aos objetivos do processo de gerência de riscos definido. Estes componentes são sugestões de como o processo pode ser executado. Uma organização que esteja acostumada a, por exemplo, identificar riscos utilizando a técnica Delphi, pode desenvolver um componente que implemente esta técnica, vinculando-o ao ponto de adaptação referente à identificação de riscos. Assim as equipes de projetos podem executar a atividade em questão da forma que sempre fizeram, mas utilizando a ferramenta. Foram desenvolvidos cinco componentes, descritos a seguir.

- BasicRiskManagementPlanning: este componente foi desenvolvido para auxiliar o gerente de projeto a elaborar um plano de gerência de riscos. $\mathrm{O}$ componente oferece um modelo de plano utilizado na organização. Cabe então ao gerente decidir o que aproveitar e o que mais deverá ser incluído. Se o plano sofrer alterações, o componente se encarrega de manter um registro das revisões; 
- BasicRiskIdentification: o componente de identificação de riscos permite ao gerente de projetos cadastrar os riscos do seu projeto (Figura 3). Uma vez cadastrados, os riscos podem ser monitorados durante todo o ciclo de vida;

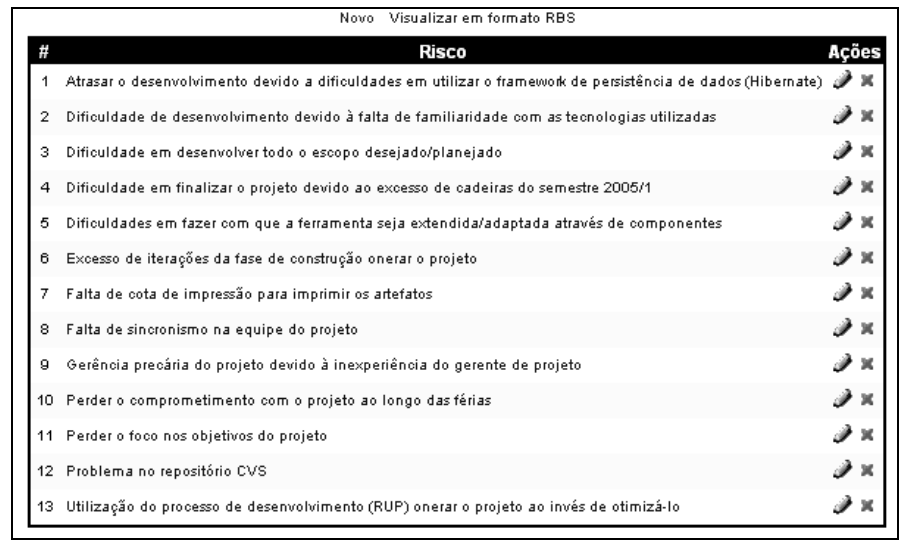

Figura 3 - Exemplo de lista de riscos gerada pela ferramenta RiskFree

- BasicRiskAnalysis: uma vez identificados os riscos do projeto, estes devem ser analisados de forma a determinar a probabilidade de ocorrência e impacto causado caso o risco venha a ocorrer. Definidas estas duas propriedades, torna-se possível priorizar os riscos identificados, com atenção especial aos principais riscos que estejam expondo o projeto a uma situação indesejada. Isto pode ser verificado através da matriz de probabilidade e impacto;

- BasicRiskResponsePlanning: para cada risco identificado deve-se planejar como este será endereçado. Isto inclui definir responsável, estratégia (mitigação, aceitação, transferência, etc.), ações previstas, ações de contingência, e outras informações que podem fazer parte do plano de resposta ao risco (Figura 4);

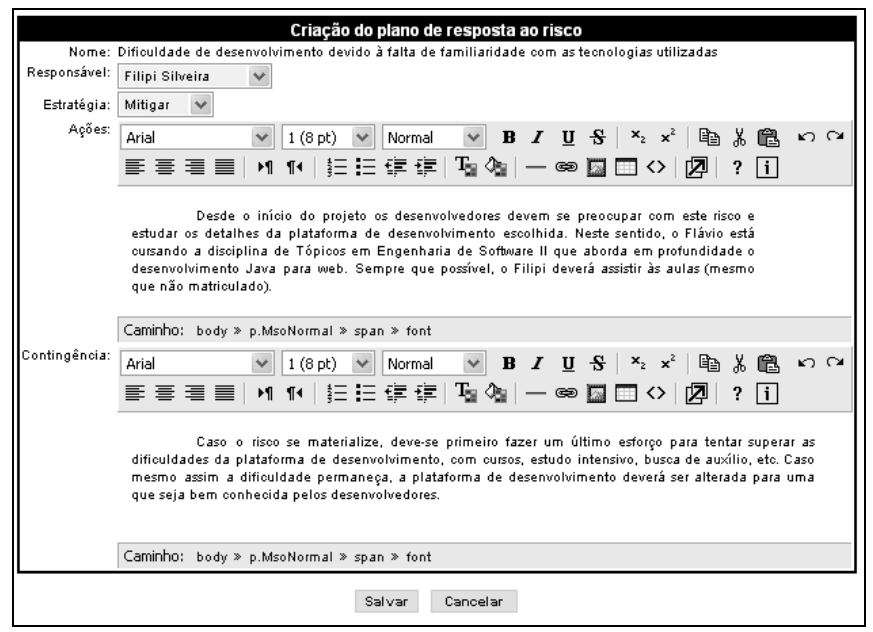

Figura 4 - Exemplo de plano de resposta a riscos na ferramenta RiskFree

- BasicRiskMonitoringAndControl:através do componente de monitoramento e controle de riscos o gerente de projeto pode acompanhar a situação dos riscos identificados e manter um registro periódico da situação de cada risco do projeto. Os componentes ficam disponíveis para os usuários através do menu de navegação, dentro do ponto de adaptação à que foram vinculados. 


\section{Considerações finais}

Acredita-se que as dificuldades de assimilação da gerência de riscos podem ser minimizadas através de uma ferramenta que facilite o trabalho dos gerentes de projetos. No entanto, muitas vezes as organizações não têm acesso a estas ferramentas devido ao seu custo elevado. Estes foram os dois principais fatores que motivaram e justificaram o desenvolvimento da ferramenta RiskFree.

A ferramenta pode ser utilizada por qualquer organização, já que não tem custo e não é dependente de outros softwares proprietários. A organização que decidir adotar a ferramenta poderá adaptá-la às suas necessidades, criando novos componentes para as etapas que compõem o processo de gerência de riscos e, por fim, a ferramenta tem como base as boas práticas de metodologias amplamente aceitas, incluindo as práticas exigidas pela área de processo de gerência de riscos do modelo CMMI (SEI, 2002).

Existem muitas oportunidades de evolução da RiskFree. O aprimoramento das funcionalidades de administração e configuração e o desenvolvimento de mais componentes são alguns dos exemplos de evoluções identificadas. Porém, acredita-se que o estágio em que a ferramenta se encontra atualmente seja suficiente para atender às necessidades de empresas iniciantes no que diz respeito à gerência de riscos. Uma vez optando por utilizar a ferramenta RiskFree, as equipes de projeto poderão: elaborar um plano de gerência de riscos; identificar riscos que afetam o projeto; analisar estes riscos com o intuito de priorizar os esforços; elaborar um plano de como o risco deverá ser controlado caso se materialize; e acompanhar as situações dos riscos do projeto.

\section{Referências bibliográficas}

Bernstein, P. (1997). Desafio aos deuses: a fascinante história do risco. Campus.

Boehm, B. (1991). Software risk management: principles and practices. Piscataway: IEEE Software, v. 8, p. 32-41.

Del Caño, A.; De La Cruz, M. P. (2002) "Integrated Methodology for Project Risk Management", Journal of Construction Engineering.

Demarco, T.; Lister, T. (2003) "Waltzing with bears: managing risk on software projects", New York: Dorset House.

Kruchten, P. (2003) “Introdução ao RUP”, Editora Ciência Moderna.

PMI (2000) "Project Management Institute - PMI: A guide to the project management body of knowledge", Syba: PMI Publishing Division, 2000.

Prikladnicki, R. et. al. (2005) "Um Caso Prático de Implantação da Gerência de Risco em Ambientes de Desenvolvimento Distribuído de Software, baseado no Modelo CMMI”. In: IV SBQS, Porto Alegre, 2005, pp: 95-102.

Schwalbe, K. (2002) “Information Technology. Project Management”, Cambridge, MA: Course Technology, 2002.

SEI (2002) "Software Engineering Institute - SEI. Capability Maturity Model Integration (CMMI) Version 1.1”, - Carnegie Mellon University, mar. 2002.

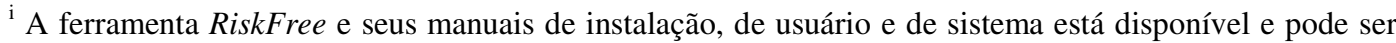
obtida no endereço http://www.inf.pucrs.br/ rafael/RiskFree/.

ii Informações sobre RiskRadar: http://www.iceincusa.com/products_tools.htm; informações sobre RiskTrack: http://www.risktrak.com; informações sobre @Risk: http://www.palisade.com/html/risk.asp.
} 